\title{
CONSUMO ALIMENTAR DE PRÉ-ESCOLARES MATRICULADOS NOS CENTROS DE EDUCAÇÃO E ALIMENTAÇÃO DO PRÉ-ESCOLAR (CEAPEs) E DE SUAS RESPECTIVAS FAMfLIAS *
}

Rosa Nilda Mazzilli **

Yaro Ribeiro Gandra**

\begin{abstract}
MAZZILLI, R. N. \& GANDRA, Y. R. Consumo alimentar de nré-escolares matriculados nos Centros de Educacão e Alimentacão do Pré-Escolar (CEAPEs) e de suas respectivas famílias. Rev. Saúde públ., S. Paulo 15(supl.):23-32, 1981.

RESUMO: Foi realizado inquórito de consumo alimentar em 383 pré- escocalares e respecttivas famílias, matriculados em 21 Centros de Educação e Alimentação do Pré-Escolar (CEAPEs) de 7 cidades do interior paulista. Utilizcu-se o método da pesagem direta dos alimentos, combinado com o recordatório, para obtenção do consumo alimentar de 24 horas da família e do pré-escolar. Concomitantemente, foram obtidas informações sobre a merenda escolar recebida e sua influência nas refeiçóes realizadas em casa pelo pré-escolar. Cerca de 50\% dos pré-escolares apresentaram deficiência energética, enquanto apenas $15 \%$ nâo alcançaram $90 \%$ ou mais de ađequação de consumo de proteínas. Os pré-escolares apresentaram ainda deficiência acentuada de vitamina A, cálcio, niacina e vitamina C. Quanto às famílias, as maiores deficiências alimentares foram em relação à vitamina $A$ e ao cálcio. A merenda escolar influenciou consumo alimentar do pré-escolar, tanto em relação ao número de refeições, quanto à quantidade de alimentos ingeridos em casa.
\end{abstract}

UNITERMOS: Pré-escolares, nutrição. Alimentos, consumo. CEAPE.

\section{INTRODUÇAO}

Todo programa de suplementação alimentar requer diagnóstico claro das condições nutricionais do grupo da população ao qual se destina. Assim, para implantar medidas tendentes a contribuir para a melhoria do estado nutricional de pré-escolares, como são os programas de suplementação alimentar, é recomendável dispor-se de informações sobre o consumo real de alimentos desse grupo etário. A assistência alimentar a esse segmento da população deveria ser prioritária, considerando-se que a qualidade e a quantidade dos alimentos ingeridos pelo pré-escolar são fatores determinantes do seu crescimento e desenvolvimento.

Muitos são os estudos já realizados sobre o consumo alimentar de familias, contudo, com referência à ingestão individual e, particularmente de pré-escolares, as pesquisas são mais escassas, sobretudo em nosso meio.

A maioria dos estudos entre pré-escolares foi levada a cabo por Flores e col, $4,5,6,7,8$.

* Convênio 10/77 - INAN/DN/FSP/USP.

** Do Departamento de Nutrição da Faculdade de Saúde Pública da USP -. Av. Dr. Arnaldo, 715 - 01255 - São Paulo, SP - Brasil. 
MAZZILLI, R.N. \& GANDRA, Y.R. Consumo alimentar de pré-escolares matriculados nos Centros de Educação e Alimentação do Pré-Escolar (CEAPEs) e de suas respectivas familias. Rev. Saúde públ., S. Paulo, 15(supl.): 23-32, 1981.

Esses autores recomendam que, ao se investigar o consumo alimentar de pré-escolares, este seja realizado concomitantemente ao da família.

Tal procedimento, embora aumente a duração do estudo e, consequientemente, o seu custo, proporcionará dados que permitirão conclusões mais confiáveis.

Em estudo longitudinal sobre a dieta da familia e do pré-escolar, em três comunidades indigenas de Guatemala, Flores e col.s verificaram que a ingestão média de nutrientes entre as crianças apresentou diferenças muito pequenas. Entretanto, quando se comparou o consumo alimentar do pré-escolar com o da familia, esses valores corresponderam, aproximadamente, a $50 \%$ daqueles encontrados para as respectivas familias.

Em outro estudo, abrangendo família e pré-escolar, realizado na área rural de $\mathrm{Pa}$ namá 4 , esses mesmos autores evidenciaram que o consumo alimentar deste último grupo segue o padrăo de sua família, variando apenas nas quantidades dos alimentos, o que confirma 0 trabalho citado anteriormente.

Para se ter idéia mais precisa do valor da suplementação alimentar fornecida, sob a forma de merenda, aos pré-escolares dos Centros de Educação e Alimentação do Pré-
-Escolar (CEAPE), levou-se a cabo levantamento do consumo alimentar desses pré-escolares e das respectivas famílias, bem como do valor nutricional da merenda servida nos CEAPEs e de sua influência sobre as refeições que o pré-escolar recebe em sua casa.

Com este levantamento objetivou-se conhecer o consumo alimentar do pré-escolar e de sua familia, identificando as deficiências alimentares de ambos os grupos e verificando qual a modificação que a merenda introduziu no consumo alimentar diário do pré-escolar.

\section{MATERIAL E METODOS}

Dependendo do número de crianças e de CEAPEs funcionando em cada cidade onde se realizou este trabalho, a população de estudo foi composta, ou do universo de pré-escolares matriculados no Programa ou de parte dele e, neste caso, a amostra foi determinada por sorteio casual.

$O$ presente estudo abrangeu 383 pré-escolares e respectivas famílias, matriculados em 21 CEAPEs de 7 localidades (Tabela 1). A escolha das cidades foi intencional, objetivando-se ter na amostra CEAPEs com diferentes horários e períodos de funcionamento.

\section{TA B E L A 1}

Distribuiçăo dos pré-escolares e respectivas familias, segundo a cidade onde residem. Estado de São Paulo, 1978/1979.

\begin{tabular}{|c|c|c|c|c|}
\hline Cidades & $\begin{array}{l}\text { No de } \\
\text { Bairros }\end{array}$ & $\begin{array}{c}\text { No de } \\
\text { CEAPEs }\end{array}$ & $\begin{array}{c}\text { Pré-escolares e } \\
\text { No }\end{array}$ & e familias \\
\hline Campinas & 2 & 2 & 77 & 20,1 \\
\hline Capivari & 2 & 2 & 20 & 5,2 \\
\hline Guaratinguetá & 1 & 2 & 28 & 7,3 \\
\hline Leme & 3 & 6 & 49 & 12,8 \\
\hline Lins & 2 & 2 & 43 & 11,2 \\
\hline Sumaré & 3 & 3 & 5.1 & 13.3 \\
\hline Suzano & 4 & 4 & 115 & 30,1 \\
\hline Total & 17 & 21 & 383 & 100 \\
\hline
\end{tabular}


MAZZILLI, R.N. \& GANDRA, Y.R. Consumo alimentar de pré-escolares matriculados nos Centros de Educação e Alimentação do Pré-Escolar (CEAPEs) e de suas respectivas famílias. Rev. Saúde públ., S. Paulo, 15(supl.): 23-32, 1981.

Os CEAPEs, em todas as cidades, foram implantados em bairros periféricos, carentes de recursos, quer de abastecimento de água e de rede de esgoto, quer de estabelecimentos comerciais para aquisição de alimentos básicos.

Para a obtenção de consumo quantitativo de alimentos utilizou-se o método da pesagem direta, combinado com o recordatório, por meio de entrevista com a mãe ou pessoa responsável pelo preparo e distribuição dos alimentos na família. Cada domicílio foi visitado durante 2 dias consecutivos para permitir o conhecimento da ingestão alimentar de $24 \mathrm{~h}$ da familia e individualmente do pré-escolar, cujos dados foram coletados concomitantemente.

A ingestão de nutrientes foi calculada com base na Tabela de Composição Quimica dos Alimentos ${ }^{17} \mathrm{e}$, para se chegar à adequação de consumo, foram usadas as recomendações da National Academy of Sciences 16. Adotou-se $90 \%$ do recomendado como limite mínimo satisfatório de consumo para todos os componentes alimentares.

\section{RESULTADOS E COMENTARIOS}

A Tabela 2 mostra os resultados da adequação média diária de consumo de calorias e de nutrientes, evidenciando que há marcada deficiência de cálcio e de vitamina $A$, exceção apenas para os pré-escolares de Guaratinguetá, cuja ingestão de equivalentes de retinol ultrapassou os $100 \%$ do recomendado para esse grupo. A inadequação de consumo para os demais nutrientes $e$ energia é bem menos acentuada, com exceção das proteinas, cujo consumo mostrou-se elevado, atingindo para toda a população, tanto de famílias como de pré-escolares, acima dos $100 \%$ das recomendaçōes. Quando esses resultados são distribuídos por faixas de adequação de consumo (Tabela 3 ), observa-se que foi bastante deficiente a ingestão de energia e de todos os nutrientes, exceção apenas para as proteinas, sendo que, para alguns deles, 50 a $90 \%$ dos pré-escolares investigados apresentaram baixo consumo. Observa-se também que foi menor o percentual de famílias com baixa ingestão de energia, tiamina, niacina, cálcio, fósforo e ferro, levando à suposição de que a alimentação do pré-escolar não é fator prioritário, uma vez que a situação alimentar das familias mostrou-se melhor, tanto para energia como para cinco dos nove nutrientes analisados.

A ingestão de proteínas foi satisfatória para a maioria das familias e dos pré-escolares, 83 e $85 \%$, respectivamente (Tabela 3 ). Estes resultados concordam com os de Alves ${ }^{1}$, em seu estudo realizado no município de São Paulo, o qual mostrou que a deficiência energética é mais frequiente que a protéica.

Deve ser salientada a ingestão insatisfatória de niacina (Tabela 2), nutriente em que cerca de $65 \%$ das familias e $81 \%$ dos pré-escolares estão abaixo dos limites considerados satisfatórios. Em pesquisas sobre consumo alimentar, realizadas em várias cidades do interior paulista, verificou-se também baixa ingestão de cálcio e de vitamina $A$, enquanto que para energia $e$ os demais nutrientes a adequação de consumo foi satisfatória 2,12,13,14,15.

Analisando-se a Tabela 4, constata-se que os produtos lácteos, as frutas, as hortaliças e os ovos, importantes fontes de cálcio, riboflavina, vitamina $\mathrm{A}$, vitamina $\mathrm{C}$, tiamina e ferro, são justamente aqueles alimentos consumidos em quantidades relativamente baixas para permitirem níveis satisfatórios desses nutrientes. Nota-se que, embora o "per capita" de carne seja aceitável e o de leguminosas, praticamente representado pelo feijão, tenha sido bastante razoável, ainda assim não foi suficiente para permitir aos grupos estudados que alcançassem meIhor adequação de niacina. 


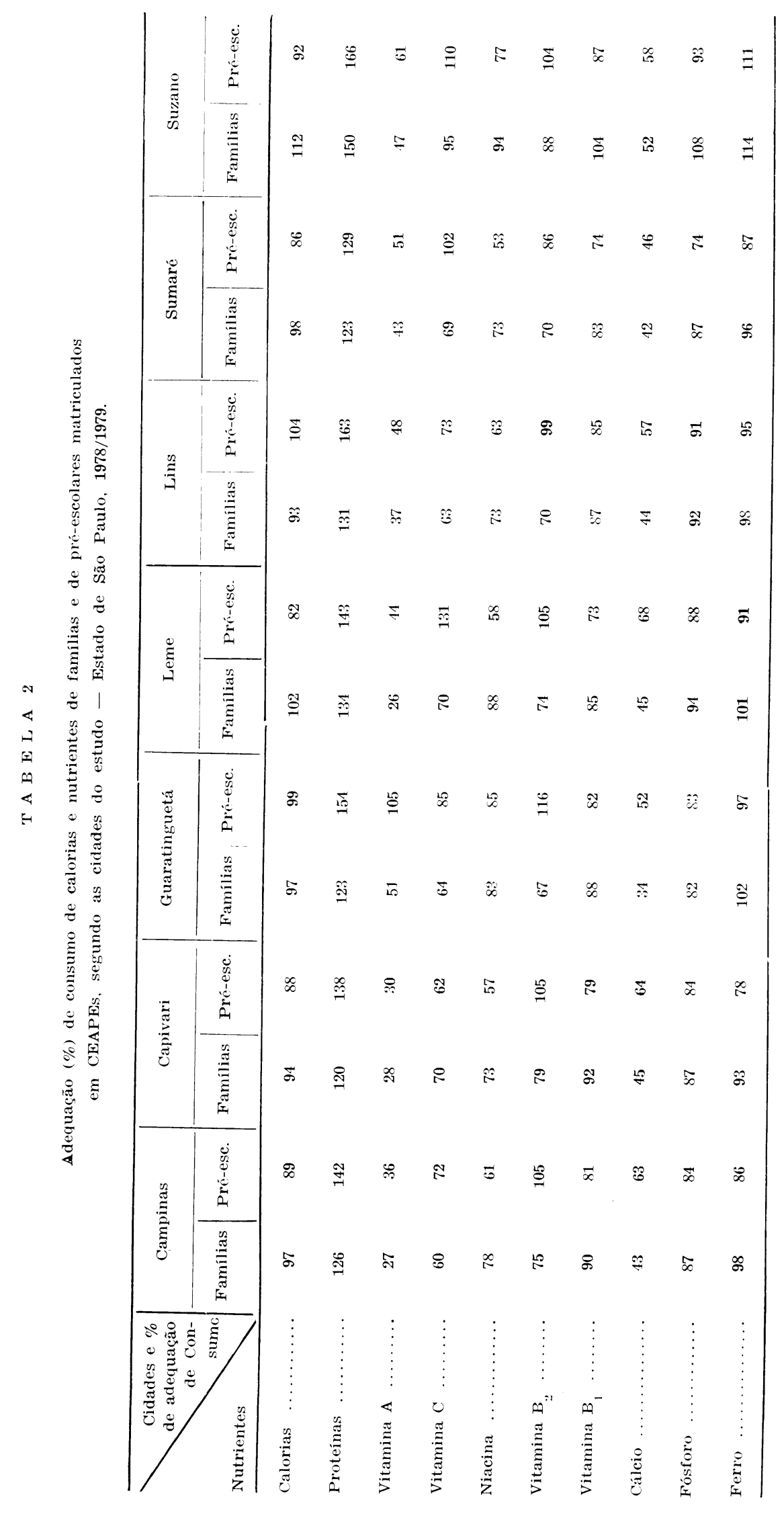




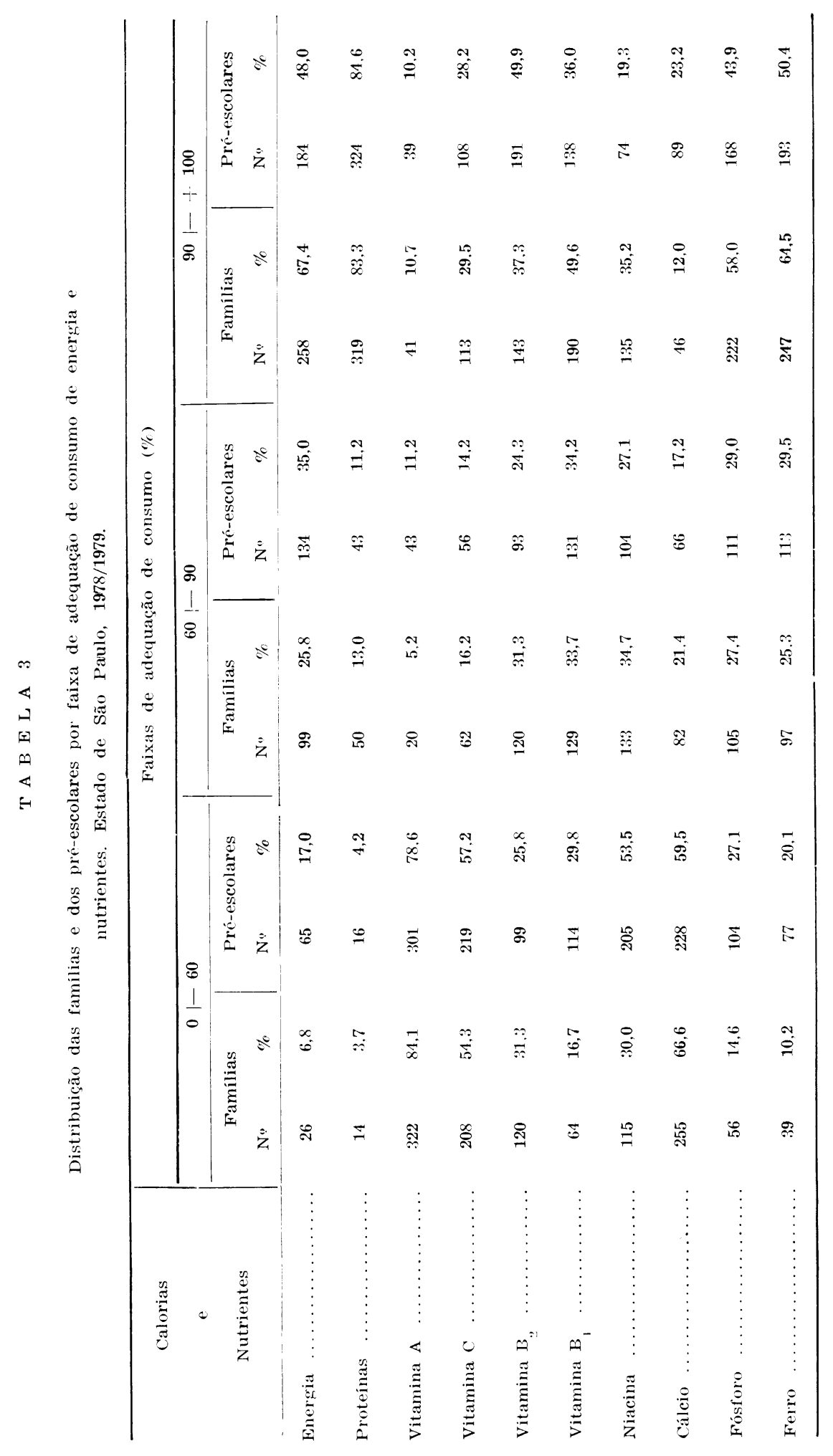




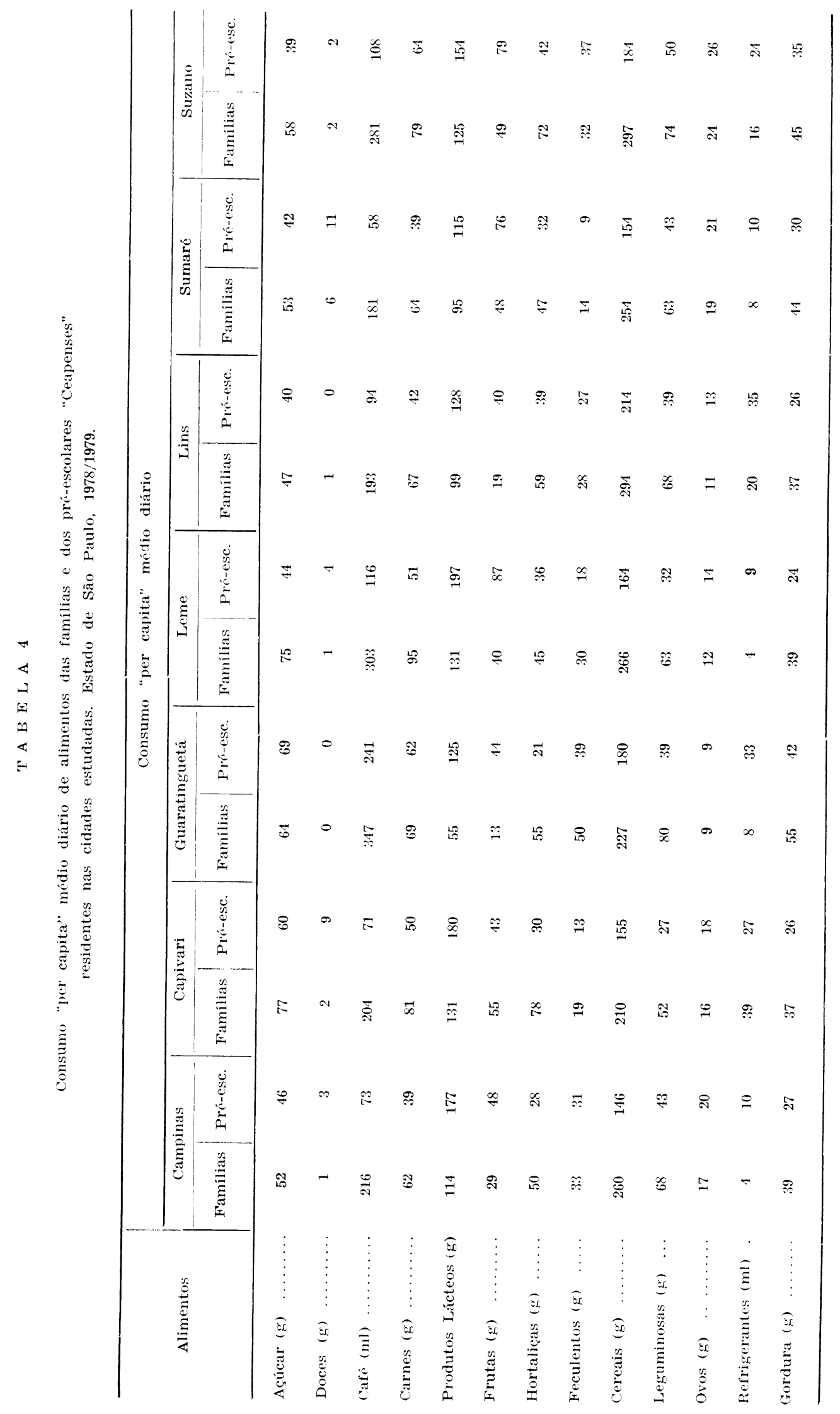


MAZZILLI, R.N. \& GANDRA, Y.R. Consumo alimentar de pré-escolares matriculados nos Centros de Educação e Alimentação do Pré-Escolar (CEAPEs) e de suas respectivas famílias. Rev. Saúde públ., S. Paulo, 15(supl.): 23-32, 1981.

A situação alimentar encontrada nos levantamentos realizados em várias cidades do interior do Estado 2,12,13,14,15, é semelhante àquela verificada no presente estudo (Tabela 2), porém difere bastante daquela observada pelo Estudo Nacional de Despesa Familiar $(E N D E F)^{9}$, para a Região São Paulo, cujos resultados mostraram ingestão mais elevada de niacina $(233 \%)$, de cálcio $(96,3 \%)$, de vitamina A $(74,0 \%)$ e de vitamina $\mathrm{C}(231,4 \%)$.

As informações sobre a merenda escolar e sua influência nas refeições ingeridas no lar, obtidas concomitantemente com o inquérito dietético, mostraram que houve modificações no comportamento alimentar domiciliar do pré-escolar. Verificou-se, entre outros aspectos que, por receberem a merenda no CEAPE, cerca de $10 \%$ dos pré-escolares passaram a não mais almoçar e $34 \%$ deixaram de tomar lanche en suas casas. Nestes casos, a suplementação alimentar não completou, mas substituiu, com prejuizo, a alimentação do lar *.

O valor médio de energia e de nutrientes das merendas de vários dias, servidas em cada CEAPE, é apresentado na Tabela 5 . Desde que essas merendas eram de iniciativa e responsabilidade de cada município, os alimentos servidos foram fornecidos pelos órgãos competentes ou, então, adquiridos pela própria Prefeitura. Cada município oferecia, aos pré-escolares, a merenda preparada com os alimentos disponíveis no momento. Desta forma, levantamos os dados referentes às merendas distribuidas aos pré-escolares, no período de estudo do consumo alimentar da população amostrada.

$O$ valor energético e protéico das merendas difere de uma cidade para outra e é o fator determinante da maior ou menor saciedade, aspecto esse que poderia ter influenciado o comportamento do pré-escolar em relação às refeições feitas no lar. Verifica-se também que, embora o valor ener- gético médio das merendas preparadas e distribuidas nos vários CEAPEs seja aceitável (350 calorias), representando $20 \%$ do total energético para o grupo estudado, nos CEAPEs de Capivari e de Leme a contribuição da merenda foi em torno de 14 a $15 \%$ do total energético diário. Em Campinas, o valor energético médio da merenda servida, nos dois CEAPEs, foi o mais alto de todas as cidades, correspondendo a $25 \%$ do total diário recomendado para energia. A merenda de um destes CEAPEs apresentou média de 213 calorias, ou seja, $12 \%$ do total diário recomendado e, justamente nesse CEAPE, o maior percentual de crianças reside na favela próxima à Escola onde funciona o programa.

As merendas contribuíram com 33 e $35 \%$, respectivamente, de proteinas e de vitamina A para o total diário recomendado aos pré-escolares; a contribuição dos demais nutrientes variou de 9 a 25\%. Considerando-se que a suplementação alimentar deve atender a $30 \%$ das necessidades diárias de energia, proteinas, minerais e vitaminas desse grupo (II Programa Nacional de Alimentação e Nutrição) ${ }^{3}$, verifica-se que a merenda servida nos CEAPEs não atinge aquele percentual recomendado, contribuindo, apenas nas seguintes proporções:

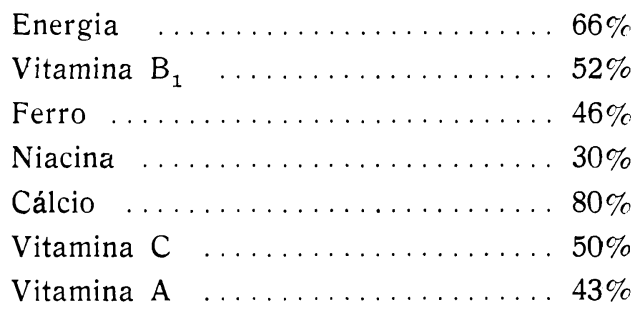

O conteúdo protéico e de vitamina $B_{2}$, entretanto, atingiram, respectivamente, 117 e $110 \%$, dos $30 \%$ previstos para esse grupo etário.

* Dados mais completos sobre as alterações das refeições domiciliares de pré-escolares que passaram a receber merenda escolar, serão publicados oportunamente. 


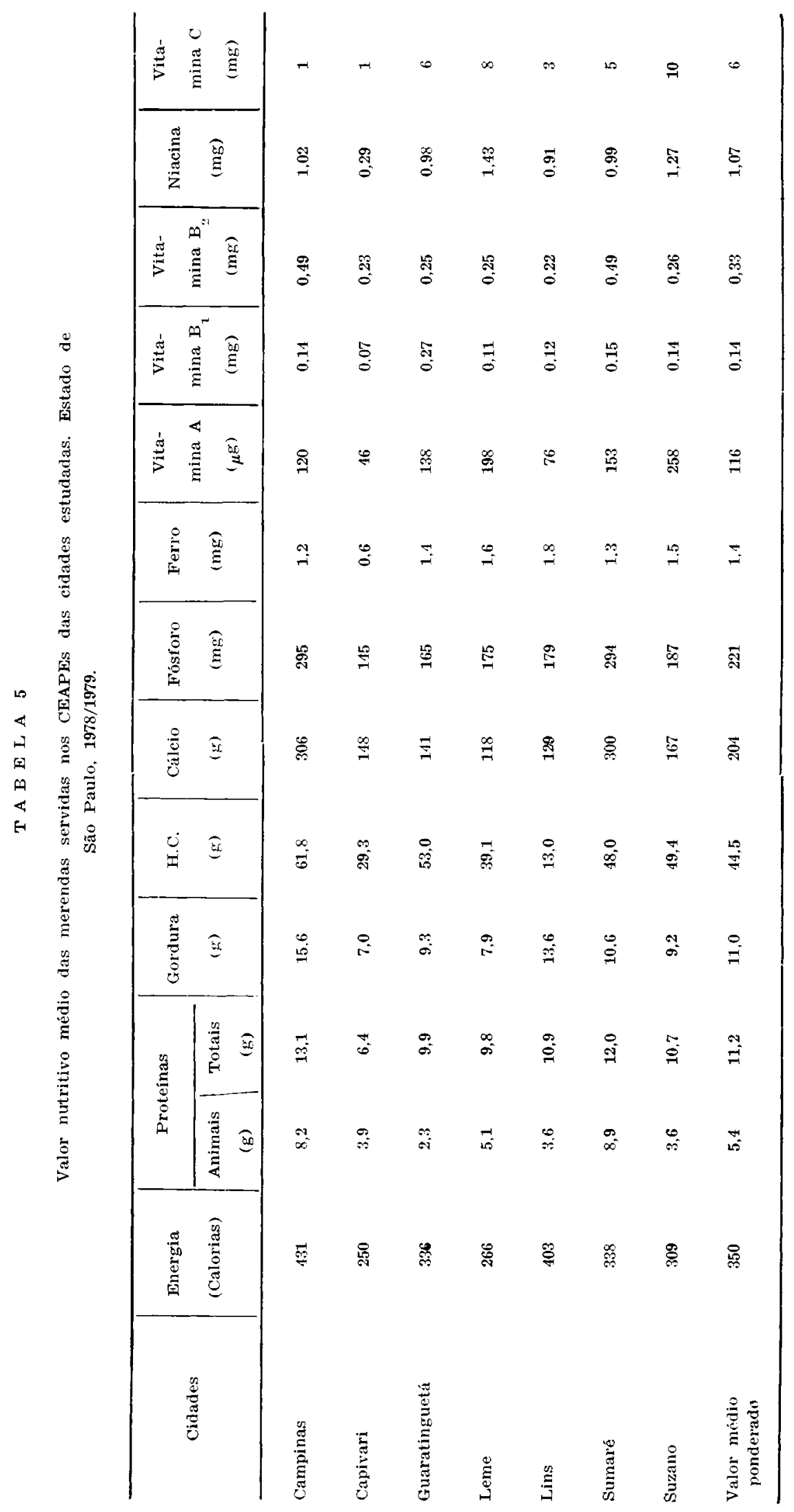


MAZZILLI, R.N. \& GANDRA, Y.R. Consumo alimentar de pré-escolares matriculados nos Centros de Educação e Alimentação do Pré-Escolar (CEAPEs) e de suas respectivas famílias. Rev. Saude públ., S. Paulo, 15(supl.): 23-32, 1981.

\section{CONCLUSOES}

Os resultados nos levam a concluir que:

-. o conteúdo energético diário da dieta foi insuficiente para, aproximadamente, $50 \%$ dos pré-escolares e $30 \%$ das familias, enquanto $83,3 \%$ das famílias e $84,6 \%$ dos pré-escolares apresentaram adequação protéica acima de $90 \%$;

- a maior deficiência encontrada foi a de vitamina $\mathrm{A}$, onde apenas $10 \%$ dos dois grupos estudados, familias e pré-escolares, atingiram $90 \%$ de adequação;

- cerca de $80 \%$ dos pré-escolares apresentaram ingestão de cálcio e niacina inferior aos $90 \%$ de adequação de consumo;

- aproximadamente $50 \%$ dos pré-escolares não alcançaram níveis satisfatórios de adequação para vitamina $B_{2}$, fósforo $e$ ferro;

- cerca de $65 \%$ das famílias não atingiram consumo adequado de niacina e vitamina $\mathrm{B}_{2}$;
- aproximadamente $90 \%$ das famílias localizaram-se nas faixas de menor adequação de cálcio;

- cerca de $70 \%$ das familias e de pré-escolares não atingiram os níveis satisfatórios de adequação de consumo de vitamina C;

- as merendas servidas nos CEAPEs contribuiram com 12 e $25 \%$ do total diário de energia recomendado para o grupo de pré-escolares do estudo;

- para parte dos pré-escolares a merenda influiu desfavoravelmente sobre a alimentação realizada no lar, tanto no que se refere ao número de refeições, quanto à quantidade de alimentos consumidos.

\section{AGRADECIMENTOS}

As docentes Marlene Trigo e Maria de Fátima Nunes Marucci, do Departamento de Nutrição da Faculdade de Saúde Pública, e aos alunos do 8 . Semestre do Curso de Graduação de Nutricionistas - Faculdade de Saúde Pública da Universidade de São Paulo, em 1979.

MAZZILLI, R. N. \& GANDRA, Y. R. [Food intake of children registered in Preschool Education and Alimeritation Centres (CEAPEs) and that of their families]. Rev. Saúde públ., S. Paulo, 15(suppl) :23-32, 1981.

ABSTRACT: A dietary survey was carried out among preschool children and their families drawn from twenty-one Preschool Education and Feeding Centre (CEAPEs), in seven towns of the State of $\mathbf{S}$. Paulo. The diatary data from children and families were obtained by applying the weighed food intake method combined with the recall method, during 24 hours, and collected by home visits. Simultaneously data on school lunches concerning their composition and influence on home meals were collected. About half the preschool children showed energy deficiency though only fifteen percent of them showed inadequate protein intake. The most outstanding dietary deficiencies in the preschool children related to vitamin A, calcium, niacin and ascorbic acid. With regard to the families, the most striking deficiencies related to vitamin $A$ and calcium. The school lunch showed some influence on the dietary intake of children, insofar as number of meals and quantity of food eaten at home were concerned.

UNITERMS: Child, preschool. Nutrition. Food. CEAPE. 
MAZZILLI, R.N. \& GANDRA, Y.R. Consumo alimentar de pré-escolares matriculados nos Centros de Educaçáo e Alimentação do Pré-Escolar (CEAPEs) e de suas respectivas famílias. Rev. Saúde públ., S. Paulo, 15(supl.): 23-32, 1981.

\section{REFERENCIAS BJBLIOGRÁFICAS}

1. ALVES, E.L.G. Nível alimentar, renda e educaçăo. Rev, ABIA/SAPRO, 30:17-44 1974.

2. BON, A.M.X. \& MIGUEL, M. O consumo de vitamina A em Ribeira, São Paulo (Brasil), Rev. Saude puibl., S. Paulo, $8: 87-92,1974$.

3. DECRETO $\mathrm{N} \otimes \mathbf{7 2 . 0 3 4}$ de 30 de Março de 1973. In: Ministério da Saúde. Legislação federal do setor saude. $2^{4} \mathrm{e}$. Brasilia, DF, Consultoria Jurídica, 1977. v. 3, p. $803-20$.

4. FLORES, M. Niveles dietéticos de famílias y niños ségun estrato-socioeconomico en el área rural de Panamá. Arch. latinoamer. Nutr., 25:135-62, 1975.

5. FLORES, M. et al. Annual of family and children's diet in three Guatemalan Indian communities. Brit. J. Nutr., 18:281-93, 1964.

6. FLORES, M. et al. Relación entre la ingesta de calorias y nutrientes en preescolares y la disponibilidad de alimentos en la familia. Aroh. latioamer. Nutr., 20:41-58, 1970.

7. FLORES, M. et al. Dieta del pre-escolar en el área rural de El Salvador. Arch. latinoamer. Nutr., 22:205-25, 1972.

8. FLORES, M. et al. Evaluación dietética de familias y preescolares mediante la aplicación de diferentes métodos y técnicas. Área rural de Nicaragua. Arch. latinoamer. Nutr., 23:325-44, 1973

9. FUNDACAO IBGE. Consumo alimentar antropometria: dados preliminares, Regiāo II - São Paulo, Região IV - Minas Gerais e Espirito Santo. Rio de Janeiro, 1977.

10. GANDRA, Y.R. Asistencia alimentaria por medio de Centros de Educación y Alimentación del Pre-Escolar. Bol. Ofic. sanit. panamer., 74:302-14, 1973.
11. GANDRA, Y.R. Nutrition and dietetic education program for pre-school children at primary school in Brazil. In: Anderson, M.A. \& Grewal, T., ed. Nutrition planning in developing world: proceedings of Regional Workshops held by CARE in India, Kenya and Colombia, 1976. Bogotá, I'rogramas Editoriales, 1976. p. 205-12.

12. MARTINS, I.S. et al. Relação entre consumo alimentar e renda familiar na cidade de Iguape, São Paulo (Brasil). Rev. Saúide públ., S. Paulo, 11:27-38, 1977 .

13. MAZZILLI, R.N. Alglimas consideraçōes sobre o consumo alimentar em Icapara e Pontal de Ribeira, São Paulo (Brasil). Rev. Saúde públ., S. Paulo, 9:49-55, 1975

14. MIGUEL, M. Resultados do inquérito alimentar realizado nas cidades de Apiai, Ribeira e Barra do Chapéu (São Paulo, Brasil), Rer. Saúde públ., S. Paulo, $8: 75-86, \quad 1974$

15. RONCADA, M.J. et al. Hipovitaminose A em comunidades do Estado de São Paulo, Brasil. Rev. Saúde públ., S. Paulo, $15: 338-49,1981$.

16. UNITED STATES. National Academy of Sciences. Recommended dietary allowances: report. $7^{\text {th }}$ ed. Washington, D.C., 1968.

17. UNIVERSIDADE DE SAO PAULO. Faculdade de Saúde Pública. Departamento de Nutrição. Tabela de Composiçāo Quimica dos Alimentos: compilaçāo de varias tabelas nacionais e estrangeiras São Paulo, 1971. [Mimeografado]

Recebido para publicasão em 10/0\%/1981

Aprovado para publicasão em 17/11/1981 